\title{
Monoclonal antibodies raised against Guillain-Barré syndrome-associated Campylobacter jejuni lipopolysaccharides react with neuronal gangliosides and paralyze muscle-nerve preparations
}

\author{
Carl S. Goodyear, ${ }^{1,2}$ Graham M. O’Hanlon, ${ }^{1}$ Jaap J. Plomp,, ${ }^{3,4}$ Eric R. Wagner, ${ }^{1}$ \\ Ian Morrison, ${ }^{1}$ Jean Veitch, ${ }^{1}$ Lynne Cochrane, ${ }^{1}$ Roland W. M. Bullens, $, 3,4$ \\ Peter C. Molenaar, ${ }^{4}$ Joe Conner, ${ }^{2}$ and Hugh J. Willison ${ }^{1}$ \\ ${ }^{1}$ University Department of Neurology, Southern General Hospital, Glasgow G51 4TF, Scotland \\ ${ }^{2}$ Department of Biological Sciences, Glasgow Caledonian University, Glasgow G4 OBA, Scotland \\ ${ }^{3}$ Department of Neurology, and \\ ${ }^{4}$ Department of Physiology, Leiden University Medical Centre, 2300 RC Leiden, the Netherlands \\ Address correspondence to: Hugh J. Willison, University Department of Neurology, Institute of Neurological Sciences, \\ Southern General Hospital, Glasgow G51 4TF, Scotland. Phone: 44-141-201-2464; Fax: 44-141-201-2993; \\ E-mail: gora13@udcf.gla.ac.uk. \\ Received for publication March 17, 1999, and accepted in revised form July 19, 1999.
}

\begin{abstract}
Guillain-Barré syndrome and its variant, Miller-Fisher syndrome, are acute, postinfectious, autoimmune neuropathies that frequently follow Campylobacter jejuni enteritis. The pathogenesis is believed to involve molecular mimicry between sialylated epitopes on C. jejuni LPSs and neural gangliosides. More than $90 \%$ of Miller-Fisher syndrome cases have serum anti-GQ1b and anti-GT1a ganglioside antibodies that may also react with other disialylated gangliosides including GD3 and GD1b. Structural studies on LPS from neuropathy-associated C. jejuni strains have revealed GT1a-like and GD3like core oligosaccharides. To determine whether this structural mimicry results in pathogenic autoantibodies, we immunized mice with GT1a/GD3-like C. jejuni LPS and then cloned mAb's that reacted with both the immunizing LPS and GQ1b/GT1a/GD3 gangliosides. Immunohistology demonstrated antibody binding to ganglioside-rich sites including motor nerve terminals. In ex vivo electrophysiological studies of nerve terminal function, application of antibodies either ex vivo or in vivo via passive immunization induced massive quantal release of acetylcholine, followed by neurotransmission block. This effect was complement-dependent and associated with extensive deposits of IgM and C3c at nerve terminals. These data provide strong support for the molecular mimicry hypothesis as a mechanism for the induction of cross-reactive pathogenic anti-ganglioside/LPS antibodies in postinfectious neuropathies.
\end{abstract}

J. Clin. Invest. 104:697-708 (1999).

\section{Introduction}

Guillain-Barré syndrome (GBS) is an acute, paralytic neuropathy with an incidence of 1-2 per 100,000 that leaves $20 \%$ of patients disabled or dead 1 year after onset (1). In the Miller-Fisher syndrome (MFS) variant of GBS, paralysis is confined to the extraocular and bulbar muscles (2). The clinical features of GBS and MFS may co-occur in any given patient to produce an overlap syndrome (3). Both GBS and MFS are postinfectious syndromes, occurring 10-14 days after diverse bacterial and viral infections, particularly Campylobacter jejuni enteritis (4). More than $90 \%$ of MFS cases and GBS overlap cases have acute-phase antibodies to GQ1b and GT1a gangliosides that disappear with clinical recovery (5). In addition, MFS sera may also contain antibodies that react with structurally similar gangliosides containing disialosyl residues, including GD3, GD1b, and GT1b (6).

Gangliosides are glycosphingolipids comprising a ceramide moiety embedded in the lipid bilayer and a sialylated oligosaccharide core that is extracellularly displayed and capable of acting as an autoantibody target (7). Gangliosides are highly enriched in distinct regional patterns within the nervous system; in particular, GQ1b is concentrated in extraocular nerves, the principal motor site affected in MFS (8).

Structural and serological studies have shown that the LPS core oligosaccharides (core OSs) of C. jejuni isolates from GBS and MFS cases can mimic gangliosides (9). Several C. jejuni isolates from neuropathy cases contain GT1a- and GD3-like structures on their core OSs $(10,11)$ that also exhibit serological cross-reactivity with gangliosides $(12,13)$. These data suggest that antiganglioside antibodies in post-C. jejuni GBS may arise through molecular mimicry. Equally, T cell-mediated attack on peripheral nerve may lead to inflammatory destruction of neural tissue as occurs in the principal animal model of GBS, experimental allergic neuritis, 
with subsequent induction of anti-ganglioside antibodies as a secondary event (14).

Assuming that anti-ganglioside antibodies arise through molecular mimicry, it is widely considered that they could still be disease epiphenomena. However, some in vitro electrophysiological evidence suggests they may be responsible for muscle weakness (15-17), possibly via their action on the neuromuscular junction (NMJ). Using the mouse phrenic nerve hemidiaphragm preparation as an ex vivo model for motor nerve terminal transmission, we have recently shown that antiGQ1b antibody containing sera, IgG fractions, and a cloned anti-disialosyl IgM antibody from a chronic patient with MFS exerts a complement-dependent, $\alpha$ latrotoxin-like effect at the NMJ, i.e., a temporary dramatic increase in spontaneous neurotransmitter release, followed by block of evoked release resulting in paralysis (18). These data suggest a direct pathogenic role for anti-GQ1b antibodies in MFS and GBS overlap.

In this study, we attempted to prove the molecular mimicry hypothesis by asking whether immunization with MFS- and GBS-associated C. jejuni LPS could induce antibodies reactive with gangliosides and whether such antibodies had pathogenic potential. To achieve this, we immunized mice with LPSs, demonstrated specific serological cross-reactive responses to structurally homologous gangliosides, and used these mice to clone anti-ganglioside mAb's. We then demonstrated that the mAb's were capable of binding to the nerve terminal and caused complement-mediated paralysis in the ex vivo muscle-nerve preparation, as seen with the disease-associated human antibodies, either upon in vitro incubation or after passive immunization of mice.

\section{Methods}

Immunization of mice with C. jejuni. Penner serostrains and isolates of C. jejuni with structurally defined core OSs were used as follows: the OH4382 and OH4384 isolates of the O:19 serostrain (10), the PG836 isolate of O:10 serostrain (11), and the O:4 serostrain (19) were all provided by J. Penner and D. Woodward (Center for Disease Control, Ottawa, Ontario, Canada), and the O:3 serostrain (20) was provided by A. Moran (University College Galway, Ireland). OH4382 and OH4384 were isolated in Japan from patients with GBS after C. jejuni enteritis, and PG836 was isolated in the United States from a patient with MFS after C. jejuni enteritis acquired in Puerto Rico. Bacteria were grown on blood agar plates in a microaerobic atmosphere and harvested into distilled water after 48 hours' growth. Bacteria were killed by heating at $60^{\circ} \mathrm{C}$ for 1 hour. LPS was isolated by hot phenol-water extraction, quantitated, and analyzed for purity by silver staining after SDS-PAGE and TLC (21). LPS core OS structures used in the study are shown in Figure 1.

Six- to 8-week old male or female $\mathrm{C} 3 \mathrm{H} / \mathrm{HeN}$, $\mathrm{BALB} / \mathrm{c}$, SJL, MRL, C57b/6J, and NZB mice (Harlan UK Ltd., Bicester, United Kingdom) were immunized intraperitoneally at 1 - to 3 -week intervals on 3 occasions in age- and sex-matched groups of 3 with $10^{8}$ CFUs of C. jejuni OH4384, O:4, and O:3 whole heatkilled organisms. Mice received a fourth immunization with $10 \mu \mathrm{g}$ of the corresponding purified LPS in incomplete Freund's adjuvent (IFA).

For $\mathrm{mAb}$ production, 14 male $\mathrm{C} 3 \mathrm{H} / \mathrm{HeN}$ mice were immunized subcutaneously with $50 \mu \mathrm{g}$ LPS from $\mathrm{OH} 4382, \mathrm{OH} 4384$, and O:3 in CFA and boosted with $50 \mu \mathrm{g}$ LPS in IFA on days 14,28 , and 42 . Three days before fusion, mice were given an intravenous injection of $50 \mu \mathrm{g}$ LPS in PBS.

Serial blood samples were collected at multiple time points via the tail vein and stored at $-20^{\circ} \mathrm{C}$ for measuring antibody responses to LPSs and gangliosides. All animal procedures conformed with United Kingdom Home Office and University of Glasgow institutional guidelines.

Production and purification of $m A b$ 's. Hybridomas were produced by fusion of $5 \times 10^{7}$ to $2 \times 10^{8}$ spleen cells with an equal number of OUR-1 cells, an ouabain-resistant subclone of the mouse myeloma cell line X63-Ag.653, by standard techniques (22). Colonies were selected by screening culture supernatants by ELISA against GD3 or GQ1b ganglioside, and were then cloned by limiting dilution. Relative levels of $\mathrm{mAb}$ reactivity to the panel of gangliosides were determined from ELISA titration curves over antibody concentrations of $10^{-2}$ to $10^{-5} \mathrm{mg} / \mathrm{mL}$, and the reciprocal of the antibody concentration that gave half-maximal binding (1/50\% maximal binding) was calculated as described previously (23). The mouse IgM mAb 22/18, obtained from the Developmental Studies Hybridoma Bank, University of Iowa (24), was negative for anti-ganglioside antibody activity and used as a control.

One-liter batches of culture supernatant were concentrated in the Vivaflow 200 system $(100,000 \mathrm{~mol}$ wt cut-off polyethersulfone membrane; Vivascience, Lincoln, United Kingdom). IgG and IgM mAb's were then purified on HiTrap protein G (Supelco, Bellefonte, Pennsylvania, USA) and recombinant Protein L columns (Actigen, Cambridge, United Kingdom), respectively. mAb's were analyzed for monoclonality, light chain type, and subclass by isoelectric focusing and Western blotting using standard methods and reagents as described previously (16). The concentrations of all mAb's were measured using a radial immunoglobulin diffusion kit (Binding Site, Birmingham, United King-

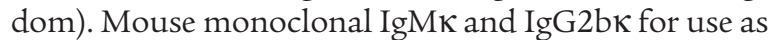
controls were obtained from Sigma Chemicals (Poole, United Kingdom).

Detection of antibodies to gangliosides and LPSs. All gangliosides (GM1, GM2, GM3, GD1a, GD1b, GD3, GT1b, GQ1b, and GA1) were supplied by Sigma Chemicals, except GT1a, which was supplied by Accurate Chemical \& Scientific Corp. (Westbury, New York, USA). GT1a is a rare ganglioside species and is not available in sufficient quantities to use as a general 
screening reagent for hybridoma production and serological screening. Accordingly, GQ1b was used in place of GT1a in many experiments, as anti-GT1a antibodies often also react with GQ1b and vice versa, although exceptions exist $(25,26)$. The basic structure of GQ1b and related gangliosides is shown in Figure 1. Mouse sera were tested for IgG and IgM response to gangliosides and LPSs by ELISA (16). Immunolon 2 microtiter plates (Dynatech, Ashford, United Kingdom) were coated with $200 \mathrm{ng}$ ganglioside in methanol or $1 \mu \mathrm{g}$ LPS in PBS per well. Each ganglioside- or LPS-coated well had a methanol- or PBS-coated control well from which background OD readings were subtracted. Plates were blocked with $2 \%$ BSA in PBS ( $\mathrm{pH}$ 7.4). Peroxidaseconjugated goat anti-mouse IgG (Fc chain-specific; Sigma Chemicals) or goat anti-mouse $\operatorname{IgM}(\mu$ chain-specific; Sigma Chemicals) was diluted 1:3,000.

TLC overlay with gangliosides or LPS was performed as described previously $(16,27)$. TLC overlays were developed using enhanced chemiluminescence (Amersham International, Amersham, United Kingdom) and scanned and digitized using the Herolab EASY scanning system (Herolab, Wiesloch, Germany). Reactivity was scored as present $(+)$ or absent $(-)$.

Immunohistological studies. Fresh unfixed tissue was dissected from the central nervous system (CNS) and peripheral nervous system (PNS) of untreated BALB/c mice and was snap-frozen in a slurry of ethanol and dry ice while embedded in Tissue-Tek OCT mounting medium (Miles Inc., Elkhart, Indiana, USA). Blocks were stored at $-70^{\circ} \mathrm{C}$ before sectioning. Cryostat sections between 5 and $15 \mu \mathrm{m}$ thick were mounted onto 3-aminopropyltriethoxysilane (APES) coated slides and allowed to air dry before use or storage at $-20^{\circ} \mathrm{C}$. Teased fiber preparations were prepared from fresh sciatic nerve using fine-gauge needles to divide the nerve into small bundles. The separated fibers were air dried onto APES-coated slides before staining. For whole-mount analysis of the diaphragm, segments of unfixed tissue were gently squashed between 2 glass slides and placed free-floating into staining medium.

Immunofluorescence labeling was performed with anti-ganglioside $\mathrm{mAb}$ or IgM/IgG control mAb concentrations of 5-7 $\mu \mathrm{g} / \mathrm{mL}$. Mounted tissue sections were incubated with $\mathrm{mAb}$ 's diluted in PBS containing $10 \%$ goat serum and $0.1 \%$ Triton X-100 overnight at $4^{\circ} \mathrm{C}$. Slides were rinsed 4 times in cold PBS and incubated with a fluorophore-labeled goat anti-mouse IgM or IgG antibody (Southern Biotechnology Associates, Birmingham, Alabama, USA) diluted 1:300 in PBS for 1 hour at $4^{\circ} \mathrm{C}$. Slides were rinsed and were then mounted in antifade solution (Citifluor, Canterbury, United Kingdom) or VECTASHIELD mounting medium with the nuclear stain DAPI (Vector Laboratories, Burlington, California, USA) to aid interpretation of nerve terminal architecture. Slides were stored at $4^{\circ} \mathrm{C}$ in the dark before viewing.

For neurofilament (NF) staining, the mouse anti-NF mAb 1217 (Affiniti Research Products, Exeter, United
Kingdom) was added to the initial incubation medium $(1: 1,000)$, and an FITC- or RITC-labeled anti-mouse IgG (1:300; Southern Biotechnology Associates) was added to the final incubation medium. The NMJ was identified by labeling the postsynaptic acetylcholine receptors with bodipy- or rhodamine-labeled $\alpha$-bungarotoxin (BTx) added to the final incubation solution (1 or $3 \mu \mathrm{g} / \mathrm{mL}$, respectively; Molecular Probes Inc., Eugene, Oregon, USA). Paranodal myelin was stained with FITC-labeled cholera toxin B subunit (CTB, 0.5 $\mu \mathrm{g} / \mathrm{mL}$; Sigma Chemicals). The complement activation product $\mathrm{C} 3 \mathrm{c}$ was identified using a FITC-labeled rabbit anti-human C3c antibody (1:300) (Dako A/S, Glostrup, Denmark) added to the final incubation solution. In passive immunization studies, the anti-IgM and anti$\mathrm{C} 3 \mathrm{c}$ antibodies already described here were used to identify IgM and C3c deposits at NMJs.

For quantification of IgM deposits at nerve terminals in passively immunized mice, unfixed diaphragm samples from 4 of the 5 control $\mathrm{mAb}$-exposed and all 4 CGM3 mAb-exposed animals were mounted in 4 blocks as pairs containing 1 of each condition. A total of $10-\mu \mathrm{m}$ sections were stained with BTx and antimouse IgM as already described. Individual NMJs were identified by their BTx staining, and, using a digital camera preset to a fixed sensitivity, they were determined as being positive if a detectable IgM signal was observed in the area colocalizing with that of BTx. The resultant data appeared to discriminate tissue from CGM3- and control IgM-treated mice in all cases after unblinding of the observer, after which the data from control IgM- and CGM3-exposed animals were statistically analyzed.

Fluorescence images were viewed via a Sony color CCD camera (Sony Corp., Tokyo, Japan) mounted on a Zeiss Axioplan microscope (Carl Zeiss Ltd., Welwyn Garden City, United Kingdom) linked to an image archiving system [Sirius VI; Optivision (Yorkshire Ltd., Ossett, United Kingdom)]. Bitmap processing and annotation were conducted on Power Point (Microsoft Corp., Redmond, Washington) and PhotoMagic and Windows Draw (both by Micrografx Inc., Richardson,

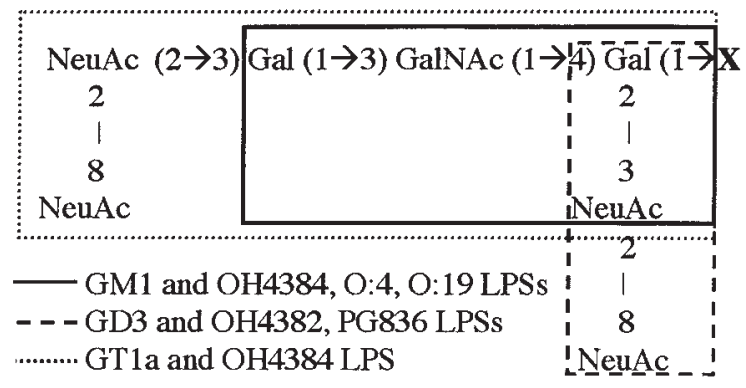

Figure 1

GQ1b and LPS core OS structures. The entire structure for GQ1b is shown. Other gangliosides and core LPSs are highlighted. NeuAc $=$ $\mathrm{N}$-acetyl neuraminic acid; $\mathrm{Gal}=$ galactose $; \mathrm{GalNAc}=\mathrm{N}$-acetyl galactosamine; $\mathrm{X}=\mathrm{Glc}(1 \rightarrow 1)$ ceramide (gangliosides) or the remaining core OS/lipid A (LPSs). 


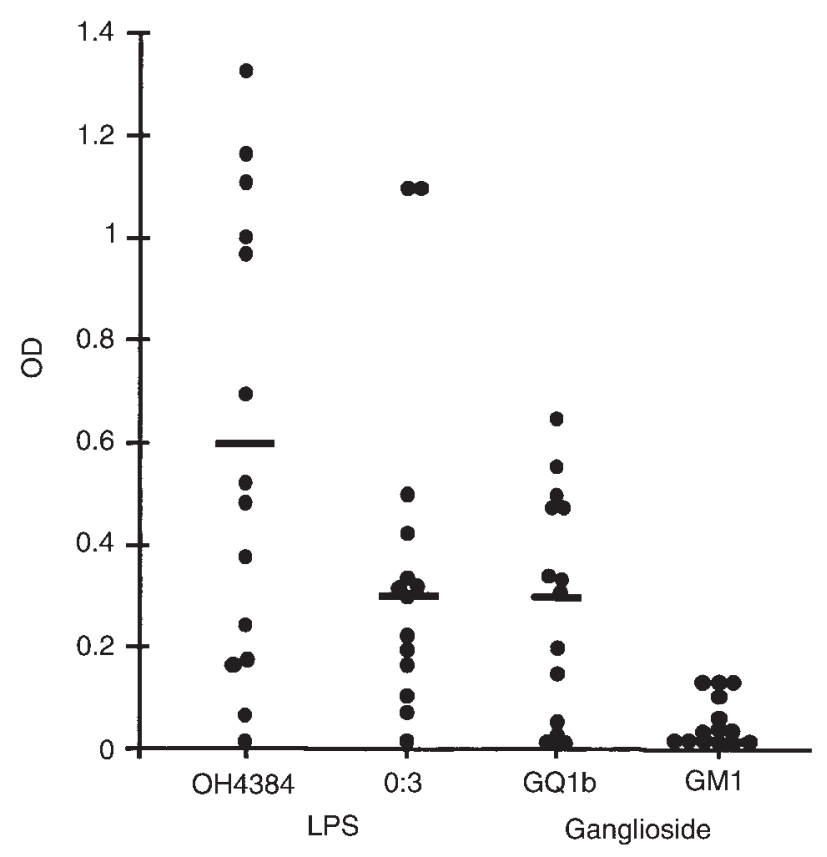

Figure 2

Serological responses to LPS and gangliosides in mice immunized with OH4384 LPS. Serum was diluted at 1:100. Filled circles represent individual animals (total $n=14$ ). Bar represents mean. Most mice elicited a serological response to the OH4384 LPS that was, on average, 2 -fold higher than the O:3 control strain (mean OD 0.60 vs. 0.27 ), which does not bear ganglioside-like LPS. In the presence of both GT1a/GQ1b-like and GM1-like epitopes on the OH4384 LPS, responder mice raised a significant antibody response to GQ1b but no response to GM1. Furthermore, in individual mice, the level of response to $\mathrm{OH} 4384$ LPS correlated highly with the level of response to GQ1b $(r=0.7 ; P=0.006)$ but not to O:3 LPS $(r=0.3 ; P=\mathrm{NS})$ or $\mathrm{GM} 1(r=0.4 ; P=\mathrm{NS})$.

Texas, USA). Microscopic images were printed directly (Kodak ColorEase; Eastman Kodak Co., Rochester, New York, USA). Controls consisting of fluorophorelabeled anti-mouse IgM alone were run in parallel with every staining batch, and the threshold of the image acquisition equipment was set so that the control level of staining was zero.

Ex vivo electrophysiology of mouse phrenic nerve hemidiaphragm preparations. Male Swiss mice (2-3 weeks old, weighing $10-20 \mathrm{~g}$ ) were sacrificed by ether inhalation according to local ethical guidelines. Left and right hemidiaphragms with phrenic nerves were dissected and pinned out in a 2-mL dish in Ringer's medium at room temperature $\left(20-22^{\circ} \mathrm{C}\right.$ ) containing (in $\mathrm{mM}$ ): $116 \mathrm{NaCl}, 4.5 \mathrm{KCl}, 1 \mathrm{MgSO}_{4}, 2 \mathrm{CaCl}_{2}, 1 \mathrm{NaH}_{2} \mathrm{PO}_{4}, 23$ $\mathrm{NaHCO}_{3}$, and 11 glucose (pH 7.4), bubbled with $95 \%$ $\mathrm{O}_{2} / 5 \% \mathrm{CO}_{2}$. Randomly within the preparation, muscle fibers were impaled near the NMJ with a 10-20 $\mathrm{M} \Omega$ glass microelectrode filled with $3 \mathrm{M} \mathrm{KCl}$. Intracellular recordings of miniature end-plate potentials (MEPPs, the small postsynaptic depolarizations resulting from spontaneous presynaptic release of single quanta of acetylcholine packaged in a single vesicle) were made at NMJs using standard recording equipment at $20-22^{\circ} \mathrm{C}$. Signals were digitized and analyzed off-line. To monitor neuromuscular transmission, the phrenic nerve was stimulated supramaximally, and the resulting contraction of the muscle was judged visually.

The mouse mAb's CGM3, CGM4, CGM5, CGG1, CGG2, and EG1 and mouse mAb myeloma IgM $\mathrm{Ind}$ IgG2bK as controls (Sigma Chemicals) were dialyzed against Ringer's medium at $4^{\circ} \mathrm{C}$ overnight before the experiments. Left hemidiaphragms were pinned out on a piece of silicone rubber and incubated in a closed vial in $1.5 \mathrm{~mL} \mathrm{mAb}$ or control IgM or IgG (at $30-80 \mu \mathrm{g} / \mathrm{mL}$ ) in Ringer's medium at $32^{\circ} \mathrm{C}$ for $3-4$ hours (to facilitate tissue penetration) followed by $4^{\circ} \mathrm{C}$ for 45 minutes (because anti-ganglioside antibody/antigen binding is facilitated at low temperature). After incubation, the mAb's or control immunoglobulin solutions were replaced by Ringer's medium, rewarmed to $20^{\circ} \mathrm{C}$, and MEPPs were then measured at 7 NMJs during a 20 -minute recording period. Subsequently, the Ringer's medium was replaced with normal human serum as a source of complement (1:1 diluted with and dialyzed overnight against Ringer's medium), and MEPPs were measured at 15 NMJs during a 30- to 45-minute recording period at $20^{\circ} \mathrm{C}$. Control MEPPs were measured on right hemidiaphragms in Ringer's at $20^{\circ} \mathrm{C}$ without any antibody preincubation.

In left hemidiaphragms from passively immunized mice (using CGM3; see later discussion), the end-plate potential (EPP, the postsynaptic depolarization resulting from acetylcholine release induced by a nerve action potential) and MEPPs were recorded after incubation of the preparations with $2.5 \mu \mathrm{M} \mu$-conotoxin GIIIB, which selectively blocks muscle sodium channels and, thus, muscle action potentials. The number of acetylcholine quanta released per nerve impulse (quantal content) at $0.3 \mathrm{~Hz}$ nerve stimulation was calculated from EPP and MEPP amplitudes (28). Also, EPP trains were recorded during about 1 second of $40 \mathrm{~Hz}$ stimulation of the nerve, and the rundown of EPP amplitude during the train was analyzed.

Passive immunization with anti-ganglioside $m A b$ 's in vivo. To determine whether mAb's bound to motor nerve terminals in vivo and then exerted clinical or electrophysiological effects, groups of mice were injected intraperitoneally with $1 \mathrm{mg} / \mathrm{d}$ of either CGM3 $(n=4)$ or the control IgM mAb 22/18 $(n=5)$ for $2-5$ days and studied 24 hours after the last $\mathrm{mAb}$ injection. One mouse from each group was studied solely by direct immunofluorescence for in vivo $\mathrm{mAb}$ deposition as follows: under halothane anesthesia, the mice were exsanguinated by cardiac puncture and the serum was retained for anti-ganglioside antibody assay. Immediately after, the diaphragm was dissected out, placed in Ringer's medium maintained at $37^{\circ} \mathrm{C}$, and rinsed 3 times over 30 minutes to wash out any unbound, extravascular IgM (the $37^{\circ} \mathrm{C}$ Ringer's-rinsed mouse). This protocol was performed to minimize the possi- 
a

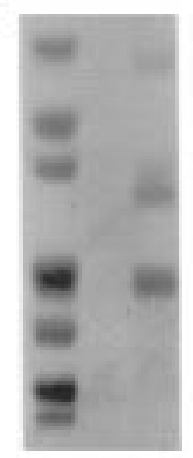

Fescreind

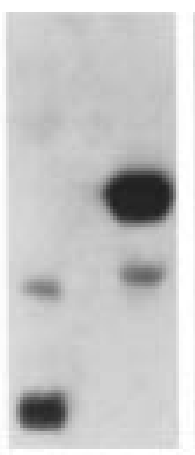

CGM1

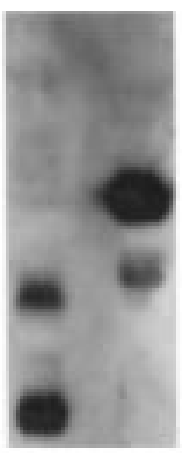

CGM2

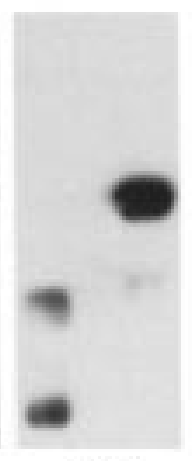

CGM3

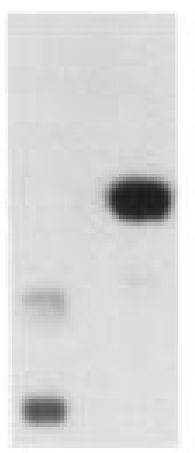

CGM4

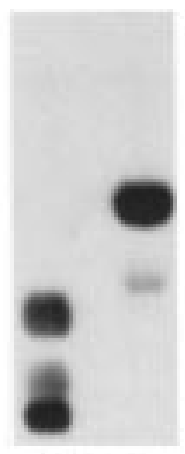

CGM5

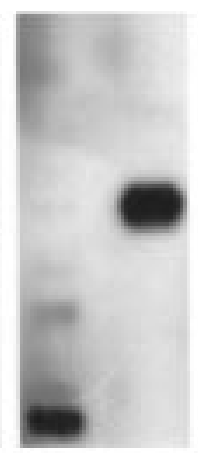

EM1

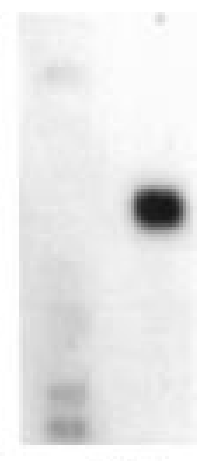

EM? b

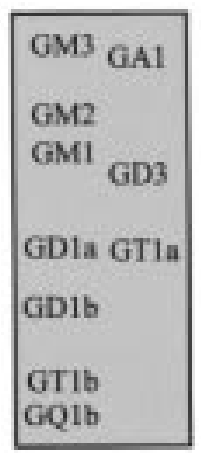

Schema

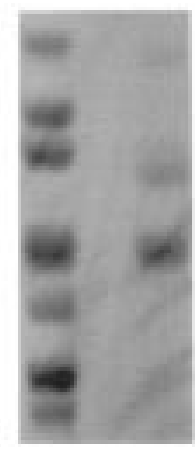

Resorcinol

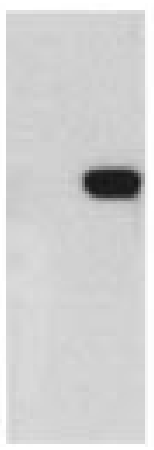

CGG1

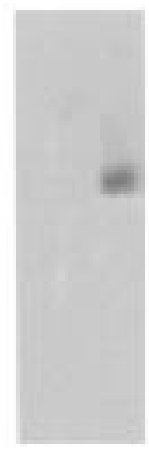

$\mathrm{CoO} 2$

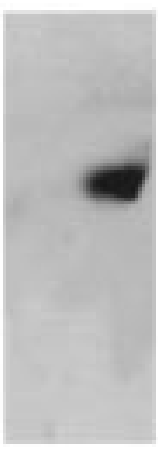

$\operatorname{coc} 3$

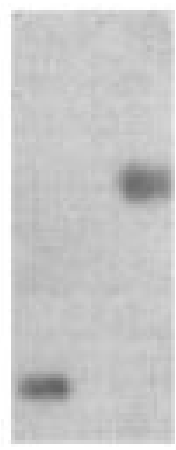

EO1 c

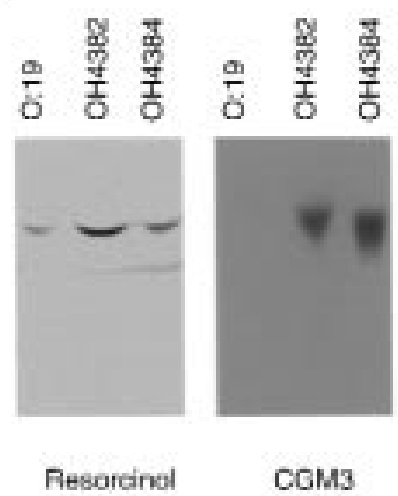

\section{Figure 3}

TLC immuno-overlay of mAb's cloned from mice immunized with LPS. The resorcinol-stained panels reveal individual ganglioside mobility, and the schema refers to both $\mathbf{a}$ and $\mathbf{b}$. All IgM mAb's (a) react strongly with GD3, and most also react with GQ1b and GT1a gangliosides. However, subtle differences in epitope specificity and intensity are evident. The $\lg G \mathrm{mAb}$ 's (b) react more selectively with GD3 only, and in the case of EG1, with GD3 and GQ1b. TLC immuno-overlay of LPSs (c) demonstrates selective reactivity of the mAb CGM3 with the immunizing GT1a-like OH4384 LPS and the structurally similar GD3-like OH4382 LPS, but not with the GM1-like O:19 serostrain LPS.

bility that $\mathrm{mAb}$ binding might have occurred at the nerve terminal during the postmortem period before immunostaining, as the CGM3 mAb-exposed mouse had a very high circulating anti-GD3 IgM antibody at a serum titer of greater than 1 per $10^{5}$. Hemidiaphragm tissue was then prepared for immunofluorescence studies as already described here. The remaining mice from each group were first analyzed electrophysiologically (as already described) and then by immunofluorescence microscopy for IgM and complement deposits.

\section{Results}

Immunization of mice with C. jejuni preparations produces specific cross-reactive responses between LPS core OSs and gangliosides. In the 6 strains of mice immunized in groups of 3 at 1- to 2-week intervals on 3 occasions with either the OH4384 or O:4 strains of heat-killed C. jejuni, the antibody response to LPSs or gangliosides was weak or absent (data not shown). After a fourth immunization with purified LPS from the corresponding C. jejuni strain, a core OS/ganglioside-specific antibody response was detected in some animals from all mouse strains. After LPS immunization, mice were often non- specifically unwell; however, no mice showed overt clinical signs of neuropathy. The data for 14 of 18 mice (4 deaths occurred as a result of endotoxic shock) immunized with OH4384 is shown in Figure 2. In preimmunization sera, anti-LPS and anti-ganglioside antibodies were undetectable (data not shown). OH4384-immunized mice elicited, on average, a 2-fold-higher serological response to OH4384 LPS when compared with O:3 LPS (which does not bear ganglioside-like epitopes; Figure 1). Serological reactivity to O:3 LPS was observed in some of the OH4384-immunized animals (mean OD at 1:100 serum dilution $=0.27 \pm 0.44 \mathrm{SD}$ ). In individual mice, however, there was no correlation between the level of OH4384 and O:3 response (Spearman's rank correlation coefficient $[r]=0.3 ; P=\mathrm{NS}$ ), indicating that any O:3 LPS response was unlikely to be cross-reactive with OH4384 LPS core OS but more likely represents an immune response to other LPS structures such as lipid A or LPS O antigen chains. This OH4384 LPS-induced O:3 LPS response was minor compared with the very high level of O:3 LPS response seen in O:3-immunized mice (mean OD after fourth immunization $=2.01 \pm 0.09 \mathrm{SD} ; n=9 / 18$ due to high mortality from endotoxic shock). The O:3-immunized 

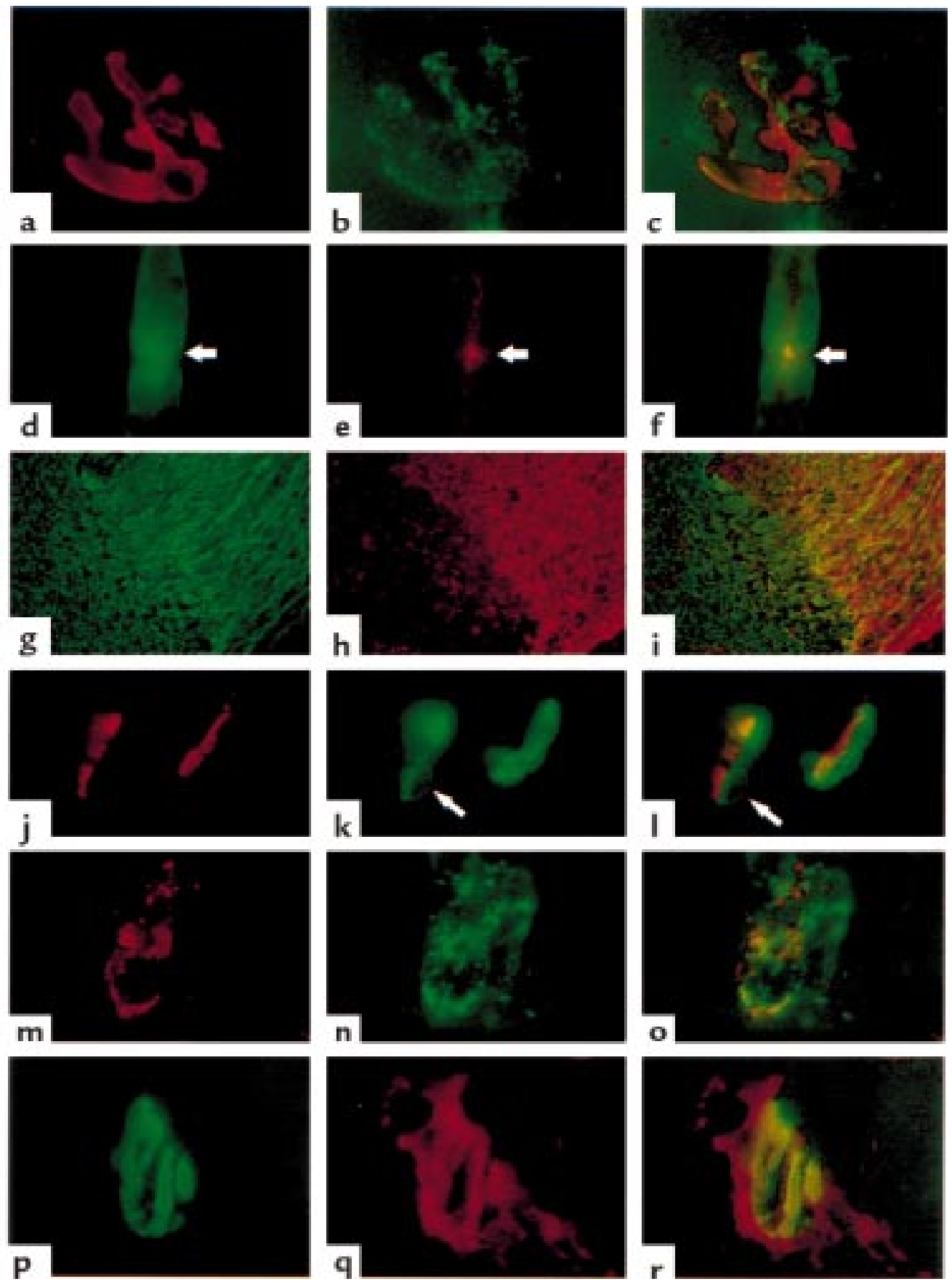

\section{Figure 4}

(a-c) Whole-mount diaphragm NMJ colabeled with BTx to identify the postsynaptic membrane (a) and the mAb CGM5 (b, overlaid in c). CGM5 gives punctate staining overlaying the postsynaptic membrane $(\times 1,020)$. ( $(\mathbf{d}-\mathbf{f})$ Sciatic nerve node of Ranvier colabeled with $C T B(\mathbf{d})$ and the $m A b$ CGM3 (e, overlaid in $\mathbf{f}$ ). CTB stains the paranodal myelin that lies either side of the nodal gap (arrow). CGM3 gives a bright signal directly overlying the nodal axolemma, with a weaker ribbon of axonal staining either side of the node $(\times 790)$. $(\mathrm{g}-\mathbf{i})$ Mouse trigeminal nerve at the transition zone between the PNS (right) and the CNS (left), colabeled with anti-NF (h) and the mAb CGM3 (h, overlaid in $\mathbf{i})$.CGM3 strongly and preferentially binds the peripheral portion of this nerve $(\times 90)$. (j-I) Transverse section through 2 NMJs from an electrophysiological preparation of hemidiaphragm treated with the mAb CGM3 and a source of human complement. Postsynaptic regions labeled with BTx (j) are associated with human C3c deposits (k); however the BTx and human C3c deposits are slightly offset (I) in a pattern suggesting that the site of complement activation is pre- rather than postsynaptic. The domed structure (arrow) is a perinuclear halo from a capping Schwann cell, indicating that complement deposits are, at least in part, on the surface of these cells $(\times 900)$. ( $\mathbf{m}-\mathbf{o})$ Whole-mount stained NMJ from an electrophysiological preparation of hemidiaphragm treated with the $\mathrm{mAb} C G M 3$ and a source of human complement. The nerve terminal contains closely but not identically overlapping deposits of IgM (m) and C3c (n, overlaid in $\mathbf{o})$. The tissue was permeabilized with detergent to expose cryptically located IgM ( $\times 960)$. (p-r) NMJ from the diaphragm of a mouse passively immunized with mAb CGM3, colabeled with BTx (p) and anti-mouse IgM (q, overlaid in $\mathbf{r})$. IgM deposits were more extensive than the area delineated by BTx $(\times 1,010)$. 
mice did not produce detectable anti-ganglioside, anti$\mathrm{OH} 4384$, or anti-O:4 LPS antibodies (data not shown).

In individual OH4384-immunized mice, the level of response to OH4384 LPS correlated highly with the level of response to GQ1b $(r=0.7 ; P=0.006)$ but not for GM1 $(r=0.4 ; P=\mathrm{NS})$ to which the response was small or absent. Thus, there is a preferential responsiveness to the GT1a-like (GQ1b cross-reactive) core OS structure rather than the GM1-like core OS structure, despite the presence of both of these epitopes on OH4384 LPS.

Ganglioside-binding characteristics of $m A b$ 's cloned from mice immunized with $\mathrm{OH} 4382$ and $\mathrm{OH} 4384$ LPSs. $\mathrm{C} 3 \mathrm{H} / \mathrm{HeN}$ mice immunized 4 times with LPS from $C$. jejuni strains $\mathrm{OH} 4382(n=3)$ or $\mathrm{OH} 4384(n=11)$ all responded with specific core OS/ganglioside antibody responses. Of the OH4382-immunized mice, 3 of 3 and 2 of 3 produced IgG and IgM serum antibodies, respectively, to GD3 (which also reacted with GQ1b and GT1a). Of the OH4384immunized mice, 9 of 11 and 4 of 11 produced $\operatorname{IgG}$ and IgM serum antibodies to GT1a (which also reacted with GD3 and GQ1b), whereas only 1 of 11 and 0 of $11(1 / 22)$ produced IgG and IgM antibodies to GM1. The 4 mice immunized with the C. jejuni O:3 control strain (which does not bear ganglioside-like moieties on its core OS) did not produce any anti-ganglioside antibody response but did produce a strong O:3 core OS response, as observed in the immunization studies already described here.

High serum antibody responders were used for cell fusion to generate mAb's. Seven IgM and $4 \operatorname{IgG}$ mAb's were cloned from 3 different mouse spleens, and their ganglioside binding characteristics are summarized in Table 1 and Figure 3. All antibodies were cloned from separate wells and had unique isoelectric mobilities, indicating that they are clonally distinct (data not shown). TLC overlay using gangliosides shows that the IgM mAb's CGM1-5 all react with GD3, GQ1b, and GT1a. In addition, for some mAb's, reactivity was observed with GD1b and an unidentified ganglioside(s) migrating between GD1a and GD1b. EM1 and EM2 have similar patterns of reactivity. The IgG mAb's CGG1-3 react exclusively with GD3, whereas EG1 reacts with both GD3 and GQ1b. None of the IgM or
IgG mAb's react significantly with monosialylated gangliosides (GM1, GM2, or GM3) or with GD1a. The relative binding of the mAb's to gangliosides were determined by titration studies in ELISA and are depicted in Table 1. Again, no mAb's reacted with monosialyated gangliosides or with GD1a (data not shown). Considering both the ELISA and TLC overlay data, the pattern of ganglioside binding of the IgM mAb's indicated a relative preference for $\alpha 2-8$-configured disialosyl epitopes $\alpha 2-3-$ linked to a terminal galactose (as found on GD3, GT1a, and GQ1b) as opposed to an internal galactose-linked disialosyl epitope (as found on GD1b and GT1b). The correlation between the relative intensities of antibody binding using ELISA and TLC overlay was in some instances poor. This is believed to be due to the influence of antigen density and orientation imposing steric constraints on the presentation of oligosaccharide in the different milieu of enzyme immunoassay plates and silica gel.

Binding of mAb's to C. jejuni LPSs shows selective reactivities to structurally bomologous core OSs. ELISA (Table 1) and TLC-overlay studies (Figure 3c) showed that all mAb's reacted with the immunizing LPS against which they were raised, either OH4382 or OH4384. Most mAb's (8/11) also reacted with the structurally similar LPS from PG836, which also bears a terminal disialosyl epitope. Five of $11 \mathrm{mAb}$ 's reacted with the $\mathrm{O}: 4$ serotype LPS. One mAb (CGG1) also reacted with the O:3 serotype LPS, which does not bear any ganglioside-like structures.

Immunolocalization of $m A b$ binding in peripheral nerve. IgM mAb's bound extensively to epitopes within the mouse CNS and PNS (Figure 4). At the region overlying the NMJ (as identified by BTx binding), IgM mAb immunolabeling was observed in a punctate pattern, suggestive of specialized subdomains on the plasma membrane of the nerve terminal or capping Schwann cells (Figure 4, a-c). In teased fiber preparations of sciatic nerve, the mAb's bound strongly to nodes of Ranvier (Figure 4, d-f). Schwann cell cytoplasmic channels were also labeled. In comparative regional studies, the mAb's labeled transverse sections of somatic nerve bun-

Table 1

Ganglioside and LPS binding characteristics of mAb's cloned from mice immunized with C. jejuni LPS

\begin{tabular}{|c|c|c|c|c|c|c|c|c|c|c|c|c|}
\hline \multirow[b]{2}{*}{ Clone } & \multirow{2}{*}{$\begin{array}{c}\text { Immunizing } \\
\text { LPS }\end{array}$} & \multirow{2}{*}{$\begin{array}{l}\text { Screening } \\
\text { Ganglioside }\end{array}$} & \multirow[b]{2}{*}{ Isotype } & \multicolumn{4}{|c|}{ Ganglioside } & \multicolumn{5}{|c|}{ C. jejuni LPS } \\
\hline & & & & GM1 & GT1a & GQ1b & GD3 & $0: 3$ & O:4 & $\mathrm{OH} 4384$ & $\mathrm{OH} 4384$ & PG836 \\
\hline CGM1 & $\mathrm{OH} 4382$ & GD3 & $\lg M \kappa$ & - & +++ & +++ & +++ & - & - & + & + & + \\
\hline CGM2 & $\mathrm{OH} 4382$ & GD3 & $\operatorname{lgM\kappa }$ & - & ++ & ++ & +++ & - & + & + & + & + \\
\hline CGM3 & $\mathrm{OH} 4384$ & GD3 & IgMк & - & ++ & +++ & +++ & - & - & + & + & + \\
\hline CGM4 & $\mathrm{OH} 4382$ & GD3 & $\lg M \kappa$ & - & ++ & +++ & +++ & - & - & + & + & + \\
\hline CGM5 & $\mathrm{OH} 4382$ & GD3 & $\operatorname{lgM\kappa }$ & - & +++ & +++ & +++ & - & - & + & + & + \\
\hline EM1 & $\mathrm{OH} 4384$ & GQ1b & IgMк & - & ++ & +++ & +++ & - & + & + & + & + \\
\hline EM2 & $\mathrm{OH} 4384$ & GQ1b & $\lg M \lambda$ & - & +++ & +++ & +++ & - & + & + & + & + \\
\hline CGG1 & $\mathrm{OH} 4382$ & GD3 & $\operatorname{lgG} 2 b \kappa$ & - & - & - & + & + & + & + & + & + \\
\hline CGG2 & $\mathrm{OH} 4382$ & GD3 & $\lg G 2 b \kappa$ & - & - & - & + & - & - & + & - & - \\
\hline CGG3 & $\mathrm{OH} 4382$ & GD3 & $\lg G 3 \kappa$ & - & - & - & + & - & - & + & - & - \\
\hline EG1 & $\mathrm{OH} 4384$ & GQ1b & $\operatorname{lgG} 3 \kappa$ & - & - & +++ & ++ & - & + & + & + & + \\
\hline
\end{tabular}




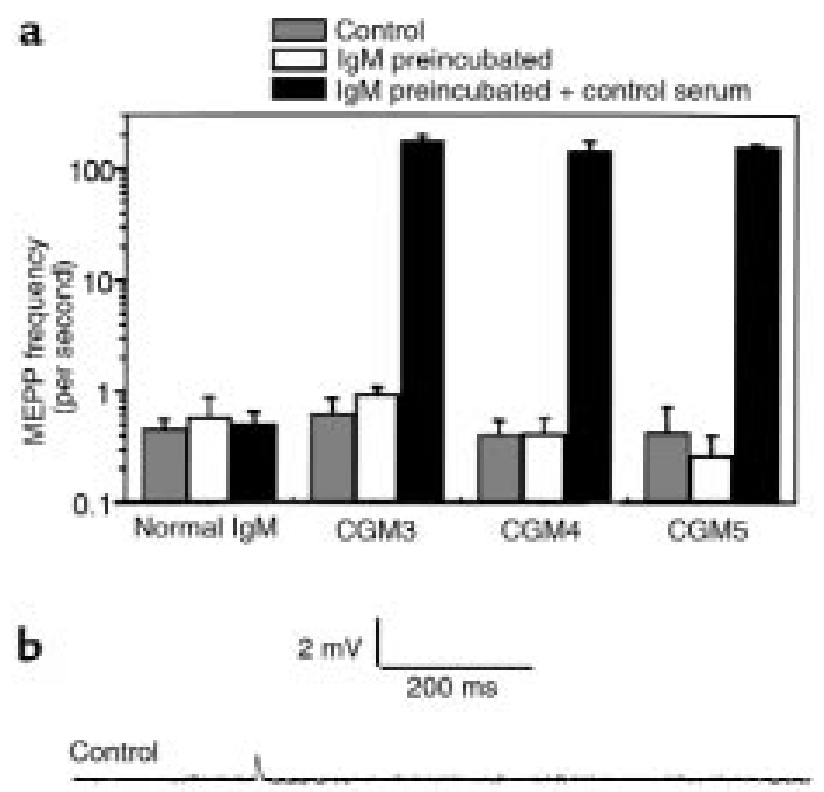

CGMS

CGM5 + Normal sorum

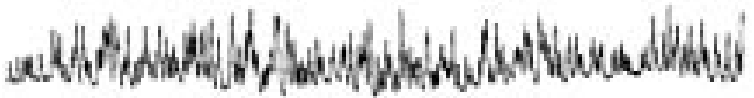

Figure 5

Effects of CGM3-5 mAb's on spontaneous quantal transmitter release at the mouse NMJ. (a) MEPP frequencies were measured in left diaphragm muscle-nerve preparations that were incubated with either CGM3 (48 $\mu \mathrm{g} / \mathrm{mL})$, CGM4 (67 $\mu \mathrm{g} / \mathrm{mL})$, CGM5 (29-39 $\mu \mathrm{g} / \mathrm{mL}$ ), or control $\operatorname{lgM}(67 \mu \mathrm{g} / \mathrm{mL})$ (open bars) and then with normal human serum as a source of complement (filled bars). In contralateral right hemidiaphragms, control MEPP frequencies were measured in Ringer's medium (shaded bars). Mean values \pm SEM ( $n$ = 2 muscles; 7-15 NMJs sampled per muscle). (b) Typical examples of 1 -second traces recorded in an untreated preparation and in CGM5-pretreated preparations with or without the addition of normal human serum as a source of complement.

dles (sciatic, phrenic) weakly compared with the intense binding observed with a cranial nerve (trigeminal). The intense trigeminal staining disappeared as the nerve entered the CNS (Figure 4, g-i). No staining above background levels was observed with any of the IgG $\mathrm{mAb}$ 's.

Application of IgM mAb's to motor nerve terminals ex vivo induces neuromuscular transmission block. The MEPP frequency of diaphragm muscle-nerve preparations treated alone with either CGM3 (48 $\mu \mathrm{g} / \mathrm{mL})$, CGM4 (67 $\mu \mathrm{g} / \mathrm{mL})$, CGM5 (29 and $39 \mu \mathrm{g} / \mathrm{mL})$, or control IgM (67 $\mu \mathrm{g} / \mathrm{mL})$ did not differ from that in untreated contralateral right hemidiaphragms $\left(\sim 0.5\right.$ seconds $\left.^{-1}\right)$ (Figure 5a). All muscles preincubated with antibody contracted normally upon supramaximal stimulation of the phrenic nerve. However, within a few minutes from the start of a subsequent incubation with normal human serum to provide complement, asynchronous twitching of muscle fibers appeared on a very large scale in the preparations preincubated with CGM3-5. After about 30 minutes, the fiber twitching subsided and large parts of the muscle became paralyzed; i.e., there was no muscle contraction visible upon stimulation of the phrenic nerve. MEPP frequency in twitching $\mathrm{mAb}$-treated preparations was extremely high $(>300$ seconds $^{-1}$ at some NMJs) compared with the mean values before the addition of normal serum (Figure 5). Occasionally, superimposed MEPPs were seen to trigger a muscle action potential. In areas of the muscle that eventually became paralyzed, MEPPs could no longer be observed. The MEPP frequency in preparations pretreated with normal control $\operatorname{IgM}(67 \mu \mathrm{g} / \mathrm{mL})$ was not affected by the incubation with normal human serum (Figure 5a), and no paralysis occurred.

The IgG mAb's EG1 $(80 \mu \mathrm{g} / \mathrm{mL})$, CGG1 $(65 \mu \mathrm{g} / \mathrm{mL})$, and CGG2 $(67 \mu \mathrm{g} / \mathrm{mL})$, as well as the control IgG (67 $\mu \mathrm{g} / \mathrm{mL}$ ), did not have effects on MEPP frequency as described for the IgM mAb's and did not induce paralysis. Mean MEPP frequencies of muscles were in the range of $0.31-0.63$ seconds $^{-1}$ in untreated right contralateral hemidiaphragms; $0.34-0.78$ seconds $^{-1}$ in left hemidiaphragms after IgG incubation; and 0.30-0.41 seconds ${ }^{-1}$ at subsequent incubation with normal control serum. Each IgG mAb was tested in 1 experiment. $m A b$ 's and the complement activation product $C 3 c$ are deposited at blocked motor nerve terminals. No overt clinical signs of muscle weakness were evident in passively immunized mice. Immunohistochemical analysis of physiologically blocked hemidiaphragm tissue, preincubated with the CGM3 $\mathrm{mAb}$ and a source of complement, showed extensive IgM and C $3 \mathrm{c}$ deposits at the NMJ. The deposits did not exactly colocalize with the postsynaptic BTx label but were slightly offset, suggesting a presynaptic location (Figure 4, j-1). Occasional domed structures, corresponding in DAPI nuclear staining studies to capping Schwann cell perinuclear haloes, were observed in these deposits (Figure 4, arrows). This suggests that complement activation occurs at least in part on the capping Schwann cell plasma membrane that envelops the motor end plate. Whole-mount diaphragm preparations required permeabilization with detergent to maximize the IgM detection, whereas the $\mathrm{C} 3 \mathrm{c}$ deposits could be readily detected in the absence of detergent. In tissue sections, both IgM and C $3 c$ could be stained without detergent. The cryptic nature of IgM deposits in intact tissue suggests that some of the IgM mAb may be internalized by the nerve terminal or surrounding cells. Although there is very close proximity and some overlap between $\mathrm{C} 3 \mathrm{c}$ and IgM deposits at the nerve terminal, it is clear that there is not an exact colocalization between the $\mathrm{C} 3 \mathrm{c}$ and IgM deposits in this region (Figure 4, m-o).

CGM3 $m A b$ is deposited at motor nerve terminals after passive immunization in vivo and subsequently mediates ex vivo electrophysiological effects on neuromuscular transmission. Immunohistological analysis of diaphragm tissue from 
4 mice passively immunized with the mAb CGM3 revealed IgM deposition at 28 of 36 (the $37^{\circ} \mathrm{C}$ Ringer's-rinsed mouse), 16 of 17,5 of 14 , and 3 of 8 (the 3 electrophysiologically studied mice) examined end plates in each of the 4 mice, respectively (52/75 end plates were positive; mean $69 \pm 5 \% \mathrm{SEM})$. In 4 control animals analyzed in parallel, 0 of 33 (the $37^{\circ} \mathrm{C}$ Ringer's-rinsed mouse), 0 of 10, 0 of 17 , and 1 of 13 (3/4 electrophysiologically studied mice) examined end plates were IgM-positive $(1 / 73$; mean $1.4 \pm 1.4 \% \mathrm{SEM})$. An example of CGM3 deposition is shown in Figure 4, p-r.

When passively immunized mouse diaphragms were placed in Ringer's buffer for electrophysiological examination in the ex vivo hemidiaphragm assay, there was no difference in EPP amplitudes ( $24.26 \pm 1.20$ vs. 23.22 $\pm 1.88 \mathrm{mV}$ ), EPP $40-\mathrm{Hz}$ rundown (plateau level was at $73.22 \pm 0.44$ vs. $73.15 \pm 1.48 \%$ of the first EPP in the train), MEPP amplitudes (1.01 \pm 0.04 vs. $0.98 \pm 0.08$ $\mathrm{mV}$ ), or quantal content ( $33.75 \pm 1.68$ vs. $32.85 \pm 1.33$ $\mathrm{U})$ between the 4 control $\mathrm{mAb}$-treated and 3 CGM3 $\mathrm{mAb}$-treated mice, respectively (means \pm SEM; 7-15 NMJs sampled per muscle). However, as shown in Figure 6, when fresh human serum (diluted 1:2, as a source of complement) was added to the organ bath, identical electrophysiological effects were observed as those recorded in preparations preincubated ex vivo with CGM3 and complement. The mean MEPP frequency in a 30- to 45-minute sampling period (15 NMJs sampled per muscle) rose dramatically in the preparations passively immunized with CGM3-treated mice (from 0.43 \pm 0.03 to $68.45 \pm 9.43$ seconds $^{-1}$ ), whereas no change was seen with the control $\mathrm{mAb}$-immunized mice (from $0.54 \pm 0.07$ to $0.52 \pm 0.05$ seconds $\left.^{-1}\right)$. After this rise in MEPP frequency seen at NMJs from CGM3-immunized mice, block of EPPs was subsequently observed (data not shown), as reported before with preparations incubated ex vivo with anti-GQ1b antibody-positive serum from a patient with MFS (18). These electrophysiological measurements show that CGM3 mAb deposited at NMJs in vivo was subsequently able to fix heterologous human complement and mediate the complement dependent $\alpha$-latrotoxin-like effects in the ex vivo preparation.

In the mice passively immunized with CGM3 and control $\mathrm{mAb}$ that were studied solely by immunohistology (i.e., the $37^{\circ} \mathrm{C}$ Ringer's-rinsed mice), extremely low levels of deposition of mouse $\mathrm{C} 3 \mathrm{c}$ were detectable at $11 \pm 18 \%$ and $3 \pm 3 \%$ examined end plates, respectively ( $P=0.19$, no significant difference), despite abundant deposits of CGM3 at approximately $70 \%$ of end plates (as shown earlier here). Furthermore, the absolute levels of mouse $\mathrm{C} 3 \mathrm{c}$ deposition were several orders of magnitude lower that the levels of human C3c observed in the CGM3 diaphragms coincubated with fresh human serum (the anti-C $3 \mathrm{c}$ antibody does not distinguish between mouse and human $\mathrm{C} 3 \mathrm{c}$ ). In assays performed to assess the bioactivity of mouse complement on CGM3 preincubated hemidiaphragms, fresh mouse serum was unable to induce the electro-

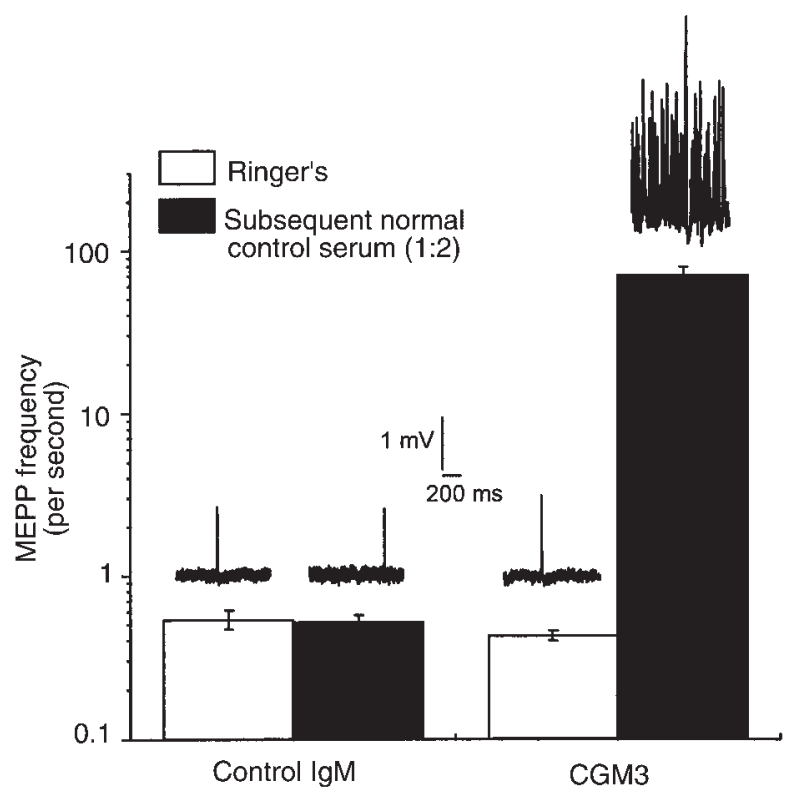

\section{Figure 6}

Effect of CGM3 mAb passive immunization on spontaneous quantal transmitter release at the mouse NMJ. Left diaphragm muscle-nerve preparations were obtained from mice treated with either CGM3 or control IgM ( $1 \mathrm{mg} / \mathrm{d}$ for 2-5 days). MEPP frequencies were measured before and during a 30 - to 45 -minute incubation of the preparations with normal human serum diluted 1:2 (filled bars). Mean values \pm SEM ( $n=3$ for CGM3 muscles, and $n=4$ for control IgM muscles; 7-15 NMJs sampled per muscle). Typical examples of 1-second traces recorded in preparations from CGM3- and control IgM-treated mice, with or without the addition of normal human serum as a source of complement, are depicted above the respective bars.

physiological blocking effect, in comparison with the positive action of fresh human and rabbit serum (data not shown). These data indicate that, in comparison with human complement, mouse complement is neither significantly activated by CGM3 antibody deposits at mouse nerve terminals in vivo nor able to mediate ex vivo blocking effects.

\section{Discussion}

Molecular mimicry between microbial and self components is widely postulated as a mechanism to account for the antigen and tissue specificity of immune responses in postinfectious autoimmune diseases (29). However, there are very few instances in which direct evidence exists, and research in this area has principally focused on $\mathrm{T}$ cell-mediated antipeptide responses, rather than humoral responses to carbohydrate structures (30). Since the original demonstration of mimicry between C. jejuni LPS and a ganglioside, this principle has been accepted as potentially relevant to the pathogenesis of GBS (9). However, proof of the pathogenic potential of LPS-induced anti-ganglioside antibodies has yet to be demonstrated, and it remains widely suggested that they may be irrelevant to the disease. This study provides further support for molecular mimicry between LPS core OS structures and gangliosides as a 
mechanism for the induction of cross-reactive pathogenic antibodies in postinfectious human neuropathies.

Our immunization studies in the mouse show that GBS patient-derived C. jejuni OH4382/4384 LPS is capable of inducing an antigen-specific response to the ganglioside-like core OS structures that cross-react with native disialylated gangliosides, e.g., GD3, GT1a, GQ1b. This principle has not been directly demonstrated in the mouse for GM1-containing C. jejuni LPSs (31) but has been demonstrated in rabbits for GM2containing LPSs and anti-GM2 antibody responses (32). Proof that the humoral response to the LPS core OS is antigen-specific is important on several counts. First, it is likely that anti-ganglioside antibodies form a component of the natural autoantibody repertoire that could be expanded nonspecifically by LPS acting as a polyclonal B-cell activator, rather than via a core OS antigen-specific B-cell receptor cross-linking (33). Indeed, the total immunoglobulin levels were markedly increased in our LPS-immunized mice (data not shown), suggesting that polyclonal activation had occurred, in addition to a core OS antigen-specific response. Our data do not preclude concurrent antibody responses to lipid A- or O-side chains, which may have antigenic sites common to many LPS species and could account for the serum antibody cross-reactivities we observed between LPSs with distinctly different core OSs. Second, the physical conformation adopted by LPS core OSs and by gangliosides, within the steric constraints imposed by their very different adjacent lipid and carbohydrate structures and epitope densities, could have prevented antigenic cross-reactivity, despite structural identity. Steric hindrance is well recognized in other studies on anti-carbohydrate antibodies (34). Third, there may be a high degree of tolerance to ganglioside-mimicking core OS structures that would prevent the induction of core OS responses that cross-reacted with self gangliosides. In the OH4384immunized mice, the preferential response to the GT1a-like, rather than the GM1-like, structure that we consistently observed, as well as the poor response to GM1-like epitopes seen by others (30), indicates that a high degree of tolerance may be elicited to the GM1like epitope in the mouse. Very high levels of specific antibody responses to the O:3 LPS (which lacks ganglioside-mimicking sialic acid and contains an unusual non-self quinolinic acid residue in its core OS structure; ref. 20) were observed in all O:3-immunized animals, suggesting that tolerance is likely to play an important role in limiting the response to core OSs that bear self carbohydrate antigens.

We confined our immunization studies to the few available GBS patient-derived C. jejuni LPSs that have been structurally defined by spectroscopic methods, so that we could definitively compare the epitope specificity of cross-reactive LPS and ganglioside antibodies. We can thus conclude that, for example, GQ1b ganglioside cross-reactivity can arise through immunization with a GT1a- or GD3-like LPS core OS structure and does not obligately require a GQ1b-like LPS structure. Furthermore, the mAb EG1, raised against the GT1a-like LPS (OH4384), reacts with GQ1b and GD3, but not GT1a. These anomalies are consistent with a previous report on $\mathrm{OH} 4382 / 4384$ showing that spectroscopic studies on LPS ganglioside-like structures correlate imperfectly with mAb-based epitope mapping studies (35). This is important when considering the host immune response repertoireto LPS, in that exposed individuals may potentially produce different patterns of anti-ganglioside antibodies after exposure to an identical microbial LPS structure.

The IgM mAb's that we cloned are all similar in their ganglioside specificity and very closely resemble the human IgM antibodies associated with a chronic MFSlike syndrome, in that they bind promiscuously to NeuNAc $(\alpha 2-8)$ NeuNAc-configured disialylated gangliosides including GD3, GT1a, and GQ1b, but not to monosialylated gangliosides such as GM1 (16). Equivalent human mAb's are found as IgM monoclonal gammopathies in patients with chronic autoimmune neuropathies with phenotypic similarities to MFS (16). We have previously shown that one such human antidisialosyl IgM mAb, termed $\mathrm{Ha} 1$, binds to many peripheral nerve sites, including motor nerve terminals (16), and blocks the mouse phrenic nerve hemidiaphragm preparation in an $\alpha$-latrotoxin-like, complement-dependent manner, as is also seen with antiGQ1b/GT1a antibody-containing MFS sera and IgG fractions (18). This electrophysiological pattern of nerve terminal block is identical to the effects we observed with CGM3-5 and further indicates that we have created LPS-induced, ganglioside cross-reactive mouse mAb's that are equivalent to our human mAb, Ha1. Furthermore, the deposition of CGM3-5 and activated complement components at the motor nerve terminal is identical to the immunodeposition pattern we have previously observed for anti-GQ1b/GT1a-containing MFS sera and $\mathrm{Ha} 1$ (18).

The antibody-induced electrophysiological effect leading to nerve terminal block in the mouse hemidiaphragm is an in vitro phenomenon that is not necessarily identical to the pathophysiology in ocular and bulbar muscles affected in MFS, although a strong resemblance is likely and a recent clinical electrophysiological case study suggests that NMJ involvement may contribute to MFS motor symptoms (36). Furthermore, clinical histopathological evidence suggests a key role for complement activation in the pathogenesis of GBS (14). There are many possible confounding factors, including the kinetics of antibody accumulation at the end plate, the ambient temperature, interspecies differences in the physiology of synaptic transmission, ganglioside distribution, and complement regulation. However, it is clear that MFS-associated anti-GQ1b/GT1a IgG antibodies, monoclonal human anti-disialosyl IgM, and the mouse mAb's described here all induce identical, complement-dependent, paralytic effects in the hemidiaphragm preparation and are thus likely to have 
a common mechanism of action in the human disease.

In contrast to the IgM mAb's, the IgG mAb's do not closely resemble their human IgG counterparts. Class switching to IgG was observed both in the serum of immunized animals and in the derived mAb's. IgG3 and IgG2b subclass mAb's were found, indicating a typical $\mathrm{T}$ cell-independent $\mathrm{TI}$ ) response as previously seen for anti-polysaccharide antibodies in the mouse (37). However, human MFS-associated anti-GQ1b IgG antibodies appear to have recruited T-cell help in that they are restricted to the $\mathrm{T}$ cell-dependent (TD) IgG1 and IgG3 subclasses, rather than the TI IgG2 subclass usually seen for human anti-carbohydrate antibodies and, as a result, may have enhanced affinity and complement fixing properties (38). In reacting with GD3 only (mAb's CGG1-3) or GD3 and GQ1b only (mAb EG1), the mouse IgG mAb's were of more selective specificity for ganglioside epitopes than the broadly reactive IgM $\mathrm{mAb}$ 's that bound many disialylated gangliosides, indicating that the IgG mAb's may have undergone a degree of epitope selection. However, there was no evidence from binding studies that their affinity or avidity was enhanced compared with the IgM mAb's. Indeed, the opposite was found, in that GD3 binding by the IgG mAb's in ELISA is weak. Furthermore, the IgG mAb's did not immunostain any neural tissue (known to contain GD3 and GQ1b) and did not block the muscle-nerve electrophysiological preparation, although explanations other than affinity-related ones are possible (34). In contrast to the highly pathogenic IgM mAb's, the murine IgG mAb's we have cloned thus differ substantially from their MFS-associated human IgG counterparts $(8,18)$.

In these studies, overt neurological disease was not present either in the LPS-immunized mice or in the animals passively immunized with the CGM3 mAb. Why these animals are refractory to the development of overt disease is not clear. In the LPS-immunized mice, the levels of specific anti-ganglioside antibody induced by immunization are relatively low and compare unfavorably both with the CGM3 passively immunized mice (serum anti-GD3 titer $>1$ per $10^{5}$ ) and with the high levels $(\sim 40 \mu \mathrm{g} / \mathrm{mL})$ of the anti-GQ1b/GT1a mAb's applied directly to the physiological assay system to induce paralysis. The lack of clinical illness in the mice is unlikely to be solely due to the level of pathogenic antibody, because we have clearly shown that sufficient antibody deposition occurs at the NMJ in vivo to produce electrophysiological malfunction ex vivo, when supplemented with a heterologous source of complement. It is thus most likely that the lack of disease in these animals is due to an absent or very low level of mouse complement activation at the nerve terminal both in vivo and ex vivo. This may be due to low levels of complement components within the NMJ, poor activation activity of murine complement (39), or inhibition by complement regulatory components and homologous restriction (40-43).
Our immunohistological studies mapping the distribution of $\mathrm{mAb}$ binding in the PNS demonstrated that in addition to binding at the NMJ, these mAb's bind many sites including dorsal root ganglia and the nodes of Ranvier of myelinated fibers, which are also affected in MFS and GBS. Recent evidence indicates that rabbits immunized with the disialylated ganglioside GD1b develop an ataxic, dorsal root ganglionopathy mediated by anti-GD1b antibodies (44). There are thus strong grounds to extend these and related studies, not only to other LPS/ganglioside cross-reactive antigens, but also to other PNS sites in addition to the NMJ.

\section{Acknowledgments}

This work was supported by grants to H.J. Willison from The Wellcome Trust and the Guillain-Barré Syndrome Support Group of Great Britain. H.J. Willison is a Wellcome Trust Senior Research Leave Fellow. J. Conner is in receipt of a Wellcome Trust New Lecturer's project grant. We are grateful to Angela Vincent (Oxford University, Oxford, United Kingdom) for critical comments on the manuscript.

1. Hahn, A.F. 1998. Guillain-Barre syndrome. Lancet. 352:635-641.

2. Fisher, M. 1956. An unusual variant of acute idiopathic polyneuritis (syndrome of ophthalmoplegia, ataxia and areflexia). N. Engl. J. Med. 225:57-65.

3. terBruggen, J.P., vanderMeche, F.G.A, deJager, A.E.J., and Polman, C.H. 1998. Ophthalmoplegic and lower cranial nerve variants merge into each other and into classical Guillain-Barre syndrome. Muscle Nerve. 21:239-242.

4. Rees, J.H., Soudain, S.E., Gregson, N.A., and Hughes, R.A.C. 1995. Campylobacter jejuni infection and Guillain Barré syndrome. N. Engl. J. Med. 333:1374-1379.

5. Chiba, A., Kusunoki, S., Shimuzu, T., and Kanazawa, I. 1992. Serum IgG antibody to ganglioside GQ1b is a possible marker of Miller-Fisher syndrome. Ann. Neurol. 31:677-679.

6. Willison, H.J., AlMemar, A., Veitch, J., and Thrush, D. 1994. Acute ataxic neuropathy with cross-reactive antibodies to GD1b and GD3 gangliosides. Neurology. 44:2395-2398.

7. Ledeen, R.W., and Wu, G. 1992. Ganglioside function in the neuron. Trends Glycosci. Glycotech. 4:174-187.

8. Chiba, A., Kusunoki, S, Obata, H., Machinami, R., and Kanazawa, I. 1997. Ganglioside composition of the human cranial nerves, with special reference to pathophysiology of Miller Fisher syndrome. Brain Res. 745:32-36.

9. Yuki, N., et al. 1993. A bacterium lipopolysaccharide that elicits Guillain Barré syndrome has a GM1 ganglioside-like structure. J. Exp. Med. 178:1771-1775.

10. Aspinall, G.O., McDonald, A.G., Pang, H., Kurjanczyk, L.A., and Penner, J.L. 1994. Lipopolysaccharides of Campylobacter jejuni serotype O:19: structures of core oligosaccharide regions from the serostrain and two bacterial isolates from patients with the Guillain Barré syndrome. Biochemistry. 33:241-249.

11. Salloway, S., et al. 1996. Miller Fisher syndrome associated with Campylobacter jejuni bearing lipopolysaccharide molecules that mimic human ganglioside GD3. Infect. Immun. 64:2945-2949.

12. Yuki, N., et al. 1994. Molecular mimicry between GQ1b ganglioside and liposaccharides of Campylobacter jejuni isolated from patients with Fisher's syndrome. Ann. Neurol. 36:791-793.

13. Jacobs, B.C., et al. 1995. Serum anti-GQ1b antibodies recognize surface epitopes on Campylobacter jejuni from patients with Miller Fisher syndrome. Ann. Neurol. 37:260-264.

14. Hartung, H.-P., et al. 1996. Autoimmune responses in peripheral nerve. Springer Semin. Immunopathol. 18:97-123.

15. Roberts, M., Willison, H.J., Vincent, A., and Newsom-Davis, J. 1994. Serum factor in Miller-Fisher variant of Guillain-Barre syndrome and neurotransmitter release. Lancet. 343:454-455.

16. Willison, H.J., et al. 1996. A somatically mutated human antiganglioside IgM antibody that induces experimental neuropathy in mice is encoded by the variable region heavy chain gene, V1-18. J. Clin. Invest. 97:1155-1164.

17. Buchwald, B., Weishaupt, A., Toyka, K., and Dudel, J. 1998. Pre-and postsynaptic blockade of neuromuscular transmission by Miller-Fisher syndrome IgG at mouse motor nerve terminals. Eur. J. Neurosci. 10:281-290. 
18. Plomp, J.J., et al. 1999. Miller Fisher anti-GQ1b antibodies: alpha-latrotoxin-like effects on motor endplates. Ann. Neurol. 45:189-199.

19. Aspinall, G.O., et al. 1993. Chemical structures of the core regions of Campylobacter jejuni serotypes O:1, O:4, O:23, and O:36 lipo-polysaccharides. Eur. J. Biochem. 213:1017-1027.

20. Aspinall, G.O., Lynch, C.M., Pang, H., Shaver, R.T., and Moran, A.P. 1995. Chemical structures of the core regions of Campylobacter jejuni O:3 lipopolysaccharide and an associated polysaccharide. Eur. J. Biochem. 213: $570-578$.

21. Wirguin, I., et al. 1994. Monoclonal IgM antibodies to GM1 and asialoGM1 in chronic neuropathies cross-react with Campylobacter jejuni lipopolysaccharides. Ann. Neurol. 35:698-703.

22. Thompson, K.M., Hough, D.W., Maddison, P.J., Melamed, M.D., and Hughes-Jones, N. 1986. The efficient production of stable human monoclonal antibody-secreting hybridomas from EBV-transformed lymphocytes using the mouse myeloma A63-Ag8.653 as a fusion partner. J. Immunol. Methods. 94:7-12.

23. Paterson, G., Wilson, G., Kennedy, P.G.E., and Willison, H.J. 1995. Analysis of anti-GM1 antibodies cloned from motor neuropathy patients demonstrates diverse variable region gene usage with extensive somatic mutation. J. Immunol. 155:3049-3059.

24. Kintner, C.R., and Brockes, J.P. 1985. Monoclonal antibodies to cells of a regenerating limb. J. Embryol. Exp. Morphol. 89:37-55.

25. O'Leary, C.P., et al. 1996. Acute oropharyngeal palsy is associated with antibodies to GQ1b and GT1a gangliosides. J. Neurol. Neurosurg. Psychiatry. 61:649-652.

26. Yuki, N., et al. 1995. Ganglioside-like epitopes of lipopolysaccharides from Campylobacter jejuni (PEN19) in three isolates from patients with Guillain Barre syndrome. J. Neurol. Sci. 130:112-116.

27. Jacobs, B.C., et al. 1997. Human IgM paraproteins demonstrate shared reactivity between Campylobacter jejuni lipopolysaccharides and human peripheral nerve disialylated gangliosides. J. Neuroimmunol. 80:23-30.

28. Plomp, J.J., van Kempen, G.T.H., and Molenaar, P.C. 1992. Adaptation of quantal content to decreased postsynaptic sensitivity at single endplates in alpha-bungarotoxin-treated rats. J. Physiol. (Lond.) 458:487-499.

29. Rose, N.R. 1998. The role of infection in the pathogenesis of autoimmune disease. Semin. Immunol. 10:5-13.

30. Karlsen, A.E., and Dyrberg, T. 1998. Molecular mimicry between nonself, modified self and self in autoimmunity. Semin. Immunol. 10:25-34

31. Wirguin, I., et al. 1997. Induction of anti-GM1 ganglioside antibodies by
Campylobacter jejuni lipopolysaccharides. J. Neuroimmunol. 78:138-142.

32. Ritter, G., et al. 1996. Induction of antibodies reactive with GM2 ganglioside after immunization with lipopolysaccharides from Campylobacter jejuni. Int. J. Cancer. 66:184-190.

33. Fearon, D.T., and Locksley, R.M. 1996. The instructive role of innate immunity in the acquired immune response. Science. 272:50-53.

34. Lloyd, K.O., Gordon, C.M., Thampoe, I.J., and Dibenedetto, C. 1992. Cell-surface accessibility of individual gangliosides in malignantmelanoma cells to antibodies is influenced by the total ganglioside composition of the cells. Cancer Res. 52:4948-4953.

35. Yuki, N., et al. 1995. Ganglioside-like epitopes of lipopolysaccharides from Campylobacter jejuni (Pen-19) in 3 isolates from patients with Guillain-Barre-syndrome. J. Neurol. Sci. 130:112-116.

36. Uncini, A., and Lugaresi, A. 1999. Fisher syndrome with tetraparesis and antibody to GQ1b: evidence for motor nerve terminal block. Muscle Nerve. 22:640-644.

37. Dullforce, P., Sutton, D.C., and Heath, A.W. 1998. Enhancement of T cell-independent immune responses in vivo by CD40 antibodies. Nat. Med. 4:88-91.

38. Willison, H.J., and Veitch, J. 1994. Immunoglobulin subclass distribution and binding characteristics of anti-GQ1b antibodies in Miller Fisher syndrome. J. Neuroimmunol. 50:159-165.

39. Ebanks, R.O., and Isenman, D.E. 1996. Mouse complement component C4 is devoid of classical pathway C5 convertase subunit activity. Mol. Immunol. 33:297-309.

40. Lazzeri, M., et al. 1998. Kidneys derived from mice transgenic for human complement blockers are protected in an in vivo model of hyperacute rejection. J. Urol. 159:1364-1369.

41. Hehmke, B., Schröder, D., Klöting, I., and Kohnert, K.D. 1991. Complement-dependent antibody-mediated cytotoxicity ( $\left.\mathrm{C}^{\prime} \mathrm{AMC}\right)$ to pancreatic islet cells in the spontaneously diabetic BB/OK rat: interference from cell-bound and soluble inhibitors. J. Clin. Lab. Immunol. 35:71-81.

42. Hänsch, G.M., Hammer, C.H., Vanguri, P., and Shin, M.L. 1981. Homologous species restriction in lysis of erythrocytes by terminal complement proteins. Proc. Natl. Acad. Sci. USA. 78:5118-5121.

43. Shen, Y., Halperin, J.A., and Lee, C.M. 1995. Complement-mediated neurotoxicity is regulated by homologous restriction. Brain Res. 671:282-292.

44. Kusunoki, S., et al. 1996. Experimental sensory neuropathy induced by sensitization with ganglioside GD1b. Ann. Neurol. 39:424-431. 